\title{
Relación entre el nivel de conocimiento y la capacidad de autocuidado en salud sexual de los adolescentes de una Institución Educativa Pública.
}

Relationship between the level of knowledge and the capacity for self-care in sexual health of adolescents in a Public Educational Institution.

Aida Milagros Vivanco Sime 1,a, Aurora Marlene Giraldo Giraldo ${ }^{1, a, c, d, e,}$, Keylla Fernanda Francia Espinoza $^{1, b}$, Catherine Helen Mescua Peñaloza ${ }^{1, b}$.

\section{RESUMEN}

Objetivo: Determinar la relación entre el nivel de conocimiento y la capacidad de autocuidado en salud sexual de los adolescentes de una Institución Educativa Pública. Material y Método: Estudio no experimental, correlacional de corte transversal. La población de estudio estuvo conformada por 262 adolescentes. Se usaron dos instrumentos que fueron adaptados y validados mediante juicio de expertos y prueba piloto en una población similar al estudio. Resultados: Predominaron los adolescentes con 16 años en 40,5\%. Respeto a las variables, predominó el nivel de conocimiento bajo en salud sexual según la dimensión social con 48,1 \%; en la dimensión biológica el 42,7\% presentó nivel de conocimiento bajo y en la dimensión psicológica el 41,2\% nivel medio. Para la capacidad de autocuidado en salud sexual en su dimensión interacción social y actividad y reposo el 35,5\% presentó capacidad de autocuidado alta y en igual porcentaje a nivel medio; sobre promoción del funcionamiento $39,7 \%$ presentaron capacidad de autocuidado alta y en bienestar personal 38,2 \% capacidad de autocuidado media. Se halló relación directa y positiva entre las variables con Rho de Spearman de 0,336 ( $\leq \leq 0,01)$. Conclusiones: Existe relación entre el nivel de conocimiento y la capacidad de autocuidado en salud sexual.

PALABRAS CLAVE: Autocuidado, conocimiento, adolescente, salud sexual.

\section{SUMMARY}

Objective: Determine the relationship between the level ok knowledge and the capacity for self-care in sexual health of adolescents in a Public Educational Institucion. Material and Methods: Non-experimental, correlational and cross-sectional study with 262 adolescents. The instruments "Knowledge in sexual health in adolescents" and "Rating Scale of the Capacity for Self-Care in Sexual Health" were adapted and validated through expert judgment and then a pilot test in a population similar to the study. Results: the majority group age is 16 years old with $40.5 \%$, respect to the variable level of knowledge in sexual health according to dimensions: social $48.1 \%$ presented low level and $18.3 \%$ high level of knowledge; biological $42.7 \%$ presented a low level and $22.9 \%$ a high level of knowledge and psychological $41.2 \%$ presented a medium level and $28.2 \%$ a high level of knowledge. Also, the capacity of self-care in sexual health in its dimensions: activity and rest $35.5 \%$ presented capacity average of selfcare; social interaction $35.5 \%$ high capacity of self-care; promotion of operation $39.7 \%$ high capacity of self-care

Facultad de Enfermería, Universidad Peruana Cayetano Heredia. Lima, Perú.

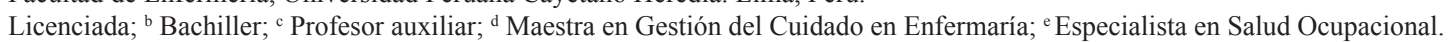


and personal well-being $38.2 \%$ medium capacity of self-care. The relationship between this variables was identified a direct and positive statistical significance with a $p \leq 0.01$ and Spearman's Rho with a score 0.336 . Conclusions: there is a relationship between the level of knowledge and the capacity for self-care in sexual health.

KEY WORDS: Self-care, knowledge, adolescent, sexual health

\section{INTRODUCCIÓN}

La adolescencia es el paso de la niñez a la adultez y comprende entre los 10 a 19 años, etapa de vida donde se atraviesan experiencias de crecimiento emocional, anatómico y fisiológico; los cuales se evidencian mediante cambios físicos, psicológicos y sociales (1), así mismo en esta etapa atraviesa situaciones y retos para el manejo de su vida y aprende pautas para afrontar su desarrollo personal.

Por su parte, la Organización Mundial de la Salud (OMS), define salud sexual como "el estado de bienestar físico, mental y social en relación a la sexualidad"(2), por lo que tanto los profesionales de la salud y la familia deben emplear un diálogo responsable sobre aspectos de salud sexual para orientar al adolescente.

Así, desde la perspectiva del profesional de la salud se deben emplear estrategias dentro y fuera del establecimiento de salud para que los adolescentes aborden la salud sexual de manera segura y oportuna(3). Por otro lado, se encuentra la familia cuya vital función es orientar hacia una sexualidad plena, previniendo situaciones de riesgo, favoreciendo estilos de vida saludable con salud integral y adecuado autocuidado frente a la salud sexual de acuerdo al Ministerio de Educación (MINEDU) según la Tutoría y Orientación Educativa "Aprendemos sobre sexualidad para orientar a nuestras hijas e hijos" (4).

El Ministerio de Salud del Perú (MINSA), desde su enfoque de la Salud Escolar tiene como objetivo "desarrollar una cultura de salud en adolescentes, de las instituciones públicas, basada en estilos de vida saludable y determinantes sociales de la salud, así como detectar y atender oportunamente los riesgos y daños relacionados con el proceso de aprendizaje" (5) como parte de las funciones que realiza el profesional de Enfermería, desde la valoración integral con un abordaje promocional - preventivo.

Asimismo, según la Estrategia Nacional de Salud Sexual y Reproductiva del Perú se menciona que estas deben ser trabajadas de manera coordinada para atender la necesidad en salud de las personas en relación a su vida sexual satisfactoria en las diversas etapas de vida (6). Por su parte la OMS propone que la salud sexual se aborde desde una perspectiva general y para este estudio prevalecerá desde el enfoque de la etapa de la adolescencia (2).

La salud sexual cuenta con la dimensión biológica compuesta por la anatomía y fisiología que expresan la sexualidad; la dimensión psicológica que abarca la independencia, responsabilidad, libertad de los adolescentes frente a sus valores morales y la dimensión social que implica vínculos afectivos y comportamientos al interactuar entre personas y entorno (7), cabe destacar que estos aspectos deberían ser de conocimiento de los adolescentes a fin de fomentar la responsabilidad sobre su salud sexual.

En este contexto, es importante que el adolescente genere su autocuidado, comprendido como el conjunto de habilidades que se aprendieron o comienzan a aprender y practicar intencionalmente. Desde la perspectiva de Orem D. en su teoría del autocuidado, afirma que las personas al interactuar con su entorno como pares, familia y escuela, reciben influencia positiva o negativa que puede repercutir en su salud e integridad; para ello se requiere la supervisión y orientación en el ejercicio del autocuidado, ya que se podría realizar de manera cotidiana sin reflexionar o tomar conciencia de la importancia de una toma de decisiones oportuna e informada en torno a la salud (8).

Por su parte, la capacidad del autocuidado (CA) en salud sexual presenta las siguientes dimensiones: Interacción social, en base al apoyo y búsqueda que realizan los adolescentes en la salud sexual; promoción del funcionamiento y desarrollo personal dentro de grupos sociales, donde se abordan hábitos, medidas y cuidados en base a la salud sexual; actividad y reposo, explorada mediante la toma de decisiones que los adolescentes realizan para su cuidado; finalmente bienestar personal, medida que pretende proteger al adolescente mediante la búsqueda y entrega oportuna de información para mejorar hábitos y actitudes en relación a la sexualidad (9). 
Ante lo expuesto por ambas variables de estudio y la experiencia sobre el trabajo comunitario que realiza el profesional de enfermería dirigida a la etapa de vida adolescente; se ve la necesidad de continuar investigando sobre esta línea de estudio.

\section{MATERIAL Y METODOS}

El estudio fue no experimental, correlacional y transversal, se ejecutó en la Institución Educativa Pública 6081 "Manuel Scorza Torres", Lima - Perú, institución escolar de nivel primaria y secundaria, situado en un distrito urbano de Lima. La población de estudio estuvo conformada por 262 adolescentes, donde se aplicaron los criterios de inclusión: estudiantes de $4^{\circ}$ y $5^{\circ}$ de secundaria, entre 14 a 17 años, cuyos padres hayan firmado el consentimiento informado y estudiantes que hayan firmado voluntariamente el asentimiento informado.

Se aplicaron cuestionarios adaptados y validados por las investigadoras mediante juicio de expertos con 10 profesionales de la salud y finalmente una prueba piloto con 50 alumnos, con características similares a la población de estudio. La técnica que se empleó para la recolección de datos fue una encuesta, ambos instrumentos fueron aplicados una vez por alumno, teniendo una duración de 20 minutos cada instrumento. El instrumento "Conocimiento en Salud de los Adolescentes", conformado por 2 items, el primero de datos demográficos del sujeto en estudio que consta de preguntas abiertas; mientras que el segundo ítem presenta preguntas cerradas de opción múltiple sobre el Nivel de conocimiento (NC) en salud sexual y su dimensión biológica, psicológica y social con 11, 7 y 9 preguntas respectivamente haciendo un total de 27 preguntas. Cabe destacar que esta propuesta se adaptó del cuestionario validado por Ricaldo en su estudio "Conocimiento sobre sexualidad de un centro educativo de Lima - proyecto de fortalecimiento, año 2006 - UNMSM” (10).

El instrumento "Escala de Valoración de la Capacidad de Autocuidado en Salud Sexual" consta de 17 preguntas según Escala de Likert, ello permite identificar la CA en las dimensiones interacción social, actividad y reposo, bienestar personal y promoción del funcionamiento y desarrollo personal dentro de grupos sociales con 4, 3, 6 y 4 preguntas respectivamente, se adaptó del instrumento validado por Isenberg y Evers en Holanda y luego traducida al español y validada por Gallegos (11).
Para la recolección de datos, se contó con la aprobación del proyecto de investigación por parte del Comité Institucional de Ética (CIE) de la UPCH, y se solicitó la autorización del Director de la Institución Educativa. Luego de realizar una reunión con los padres de familia para dar a conocer los objetivos de la investigación haciendo entrega del consentimiento informado, se dio a conocer a los adolescentes, el motivo y los objetivos de la investigación haciendo entrega del asentimiento informado.

Los datos recolectados fueron codificados e ingresaron a una base de datos creada en el programa Microsoft Excel versión 2016, donde se hizo la tabla de frecuencia absoluta, porcentaje uni y bivariadas y la gráfica. Para el cálculo se usó la prueba estadística de correlación Rho de Spearman para identificar la relación entre las variables.

Entre las limitaciones del estudio se identificaron los instrumentos, los cuales se tuvieron que adecuar en contenido y proceder con la respectiva validación.

\section{RESULTADOS}

En relación a los datos demográficos, de un total de 262 estudiantes; se tuvo similar cantidad de adolescentes varones y mujeres, donde predominó la edad de 16 años con un 40,5\% y el grado de instrucción $4^{\circ}$ de secundaria con un $53,8 \%$, según se detalla en la tabla 1.

Como resultado en la variable $\mathrm{NC}$ en salud sexual y sus dimensiones, predominó la dimensión social con nivel de conocimiento bajo en $48,1 \%$, seguido de la dimensión biológica con $42,7 \%$ en nivel de

Tabla 1. Datos demográficos de adolescentes de la Institución Educativa 6081 "Manuel Scorza Torres", Noviembre 2018.

\begin{tabular}{lcc}
\hline Datos Demográficos & n & $\mathbf{\%}$ \\
\hline Sexo & & \\
Femenino & 131 & 50,0 \\
Masculino & 131 & 50,0 \\
Edad & & \\
De 14 & 2 & 34,7 \\
De 15 & 91 & 0,8 \\
De 16 & 106 & 40,5 \\
De 17 & 63 & 24,0 \\
Grado de estudios & & \\
Cuarto grado & 141 & 53,8 \\
Quinto grado & 121 & 46,2 \\
\hline
\end{tabular}


conocimiento bajo y en la dimensión psicológica $41,2 \%$ en nivel medio, acorde a la tabla 2 .

Para la variable CA en salud sexual y sus dimensiones, se observa predominio de la dimensión Promoción del funcionamiento y desarrollo dentro de grupos sociales, la cual obtuvo nivel alto con 39,7 $\%$, seguido de bienestar personal con $38,2 \%$ en nivel medio. En interacción social predominó el nivel alto con $35,5 \%$ y en actividad y reposo el nivel medio con $35,5 \%$, acorde la tabla 3 .

Según la relación entre las variables se determinó que un $15.6 \%$ de los adolescentes presentaron un

Tabla 2. Nivel de conocimiento en salud sexual en las dimensiones biológica, psicológica y social de los adolescentes de la Institución Educativa 6081 "Manuel Scorza Torres", Noviembre 2018.

\begin{tabular}{lcccccc}
\hline \multirow{2}{*}{$\begin{array}{l}\text { Nivel de } \\
\text { conocimiento }\end{array}$} & \multicolumn{2}{c}{$\begin{array}{c}\text { Dimensión } \\
\text { biológica }\end{array}$} & \multicolumn{2}{c}{$\begin{array}{c}\text { Dimensión } \\
\text { psicológica }\end{array}$} & \multicolumn{2}{c}{$\begin{array}{c}\text { Dimensión } \\
\text { social }\end{array}$} \\
\cline { 2 - 7 } & $\mathbf{n}$ & $\mathbf{\%}$ & $\mathbf{n}$ & $\mathbf{\%}$ & $\mathbf{n}$ & $\mathbf{\%}$ \\
\hline Bajo & 112 & 42,7 & 80 & 30,5 & 126 & 48,1 \\
Medio & 90 & 34,4 & 108 & 41,2 & 88 & 33,6 \\
Alto & 60 & 22,9 & 74 & 28,2 & 48 & 18,3 \\
\hline
\end{tabular}

NC bajo y una CA media; mientras que un 4,6\% de los adolescentes presentaron un $\mathrm{NC}$ bajo y una $\mathrm{CA}$ alta y coincidentemente otro grupo de adolescentes presentaron el mismo porcentaje con $\mathrm{NC}$ alto y una CA baja, acorde la tabla 4.

Finalmente, según el Gráfico de dispersión se muestra la relación entre el $\mathrm{NC}$ y la $\mathrm{CA}$ en salud sexual, donde a mayor nivel de conocimiento existe una mayor capacidad de autocuidado, es decir que es estadísticamente directa y positiva con un valor de $\mathrm{p} \leq 0,01$, según el gráfico 1 .

\section{DISCUSIÓN}

El presente estudio tuvo como objetivo general determinar la relación entre el NC y la CA en salud sexual.

Con relación a los datos demográficos de la población de estudio, se identificó predominio de adolescentes con 16 años del $4^{\circ}$ de secundaria y en igual proporción $(50 \%)$ según género; este último resultado es similar al Censo Nacional del 2017 donde se evidencia que los adolescentes se asemejan en la cantidad de población (12). Cabe destacar, que en la

Tabla 3. Capacidad de autocuidado en salud sexual en las dimensiones Actividad y reposo, Interacción social, Promoción del funcionamiento y desarrollo personal dentro de grupos sociales y Bienestar personal de los adolescentes de la Institución Educativa 6081 "Manuel Scorza Torres", Noviembre 2018.

\begin{tabular}{lcccccccc}
\hline $\begin{array}{l}\text { Capacidad de } \\
\text { autocuidado }\end{array}$ & $\begin{array}{c}\text { Actividad } \\
\text { reposo }\end{array}$ & \multicolumn{2}{c}{ Interacción social } & $\begin{array}{c}\text { Promoción del } \\
\text { funcionamiento y } \\
\text { desarrollo dentro } \\
\text { de grupos sociales }\end{array}$ & $\begin{array}{c}\text { Bienestar } \\
\text { personal }\end{array}$ \\
\cline { 2 - 9 } & $\mathbf{n}$ & $\mathbf{\%}$ & $\mathbf{n}$ & $\mathbf{\%}$ & $\mathbf{n}$ & $\mathbf{\%}$ & $\mathbf{n}$ & $\mathbf{\%}$ \\
\hline Baja & 80 & 30,5 & 84 & 32,1 & 88 & 33,6 & 85 & 32,4 \\
Media & 93 & 35,5 & 85 & 32,4 & 70 & 26,7 & 100 & 38,2 \\
Alta & 89 & 34 & 93 & 35,5 & 104 & 39,7 & 77 & 29,4 \\
\hline
\end{tabular}

Tabla 4. Relación entre el nivel de conocimiento y la capacidad de autocuidado en salud sexual de los adolescentes de la Institución Educativa 6081 "Manuel Scorza Torres", Noviembre 2018.

\begin{tabular}{lccccccc}
\hline & \multicolumn{6}{c}{ Capacidad de autocuidado } \\
\cline { 2 - 8 } Nivel de conocimiento & \multicolumn{2}{c}{ Baja } & \multicolumn{2}{c}{ Media } & \multicolumn{3}{c}{ Alta } \\
& $\mathbf{n}$ & $\mathbf{\%}$ & $\mathbf{n}$ & $\mathbf{\%}$ & $\mathbf{n}$ & $\mathbf{\%}$ \\
\hline Bajo & 33 & 12,6 & 41 & 15,6 & 12 & 4,6 \\
Medio & 27 & 10,3 & 40 & 15,3 & 26 & 9,9 \\
Alto & 12 & 4,6 & 32 & 12,2 & 39 & 14,9 \\
Total & 77 & 29,4 & 113 & 43,1 & 72 & 27,5 \\
\hline Rs $=0,336$ & $\mathrm{p} \leq 0,01$ & & & & & &
\end{tabular}

$\mathrm{Rs}=0,336 \quad \mathrm{p} \leq 0,01$ 


\section{DE AUTOCUIDADO EN SALUD SEXUAL DE ADOLESCENTESDE UNA INSTITUCIÓN EDUCATIVA PÚBLICA}

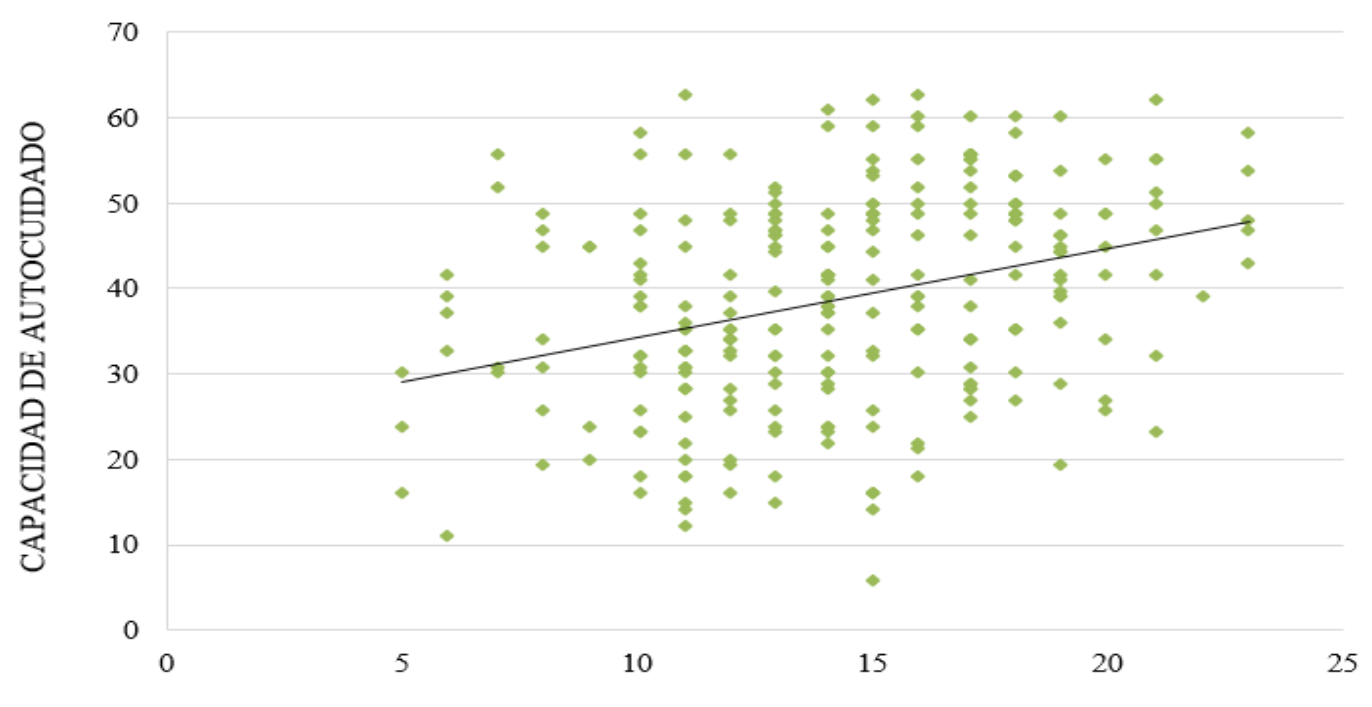

NIVEL DE CONOCIMIENTO

Gráfico 1. Tendencia de dispersión entre el nivel de conocimiento y la capacidad de autocuidado en salud sexual de los adolescentes de la Institución Educativa 6081 “Manuel Scorza Torres”, Noviembre 2018

etapa de la adolescencia, existe un notable cambio con la búsqueda de respuestas a través de un pensamiento analítico y reflexivo; siendo su desarrollo cognitivo la base para generar juicios en base a conocimientos ayudando a desarrollar su salud sexual y capacidad de autocuidado, por lo que es importante que cuenten con el apoyo y la guía de docentes y familiares por ser pilares para su educación en este proceso formativo.

Referente al NC en salud sexual y sus dimensiones, en la biológica y social obtuvieron un nivel bajo casi la mitad de adolescentes, con 42,7\% y 48,1\%; resultado similar al presentado por Dutt $\mathrm{S}$ y Manjula $\mathrm{M}$, quienes al evaluar el conocimiento sexual, actitudes y comportamientos sexuales de la juventud, encuentran relación positiva entre las variables mencionadas, y nivel de conocimiento sobre sexualidad bajo, denominado como "deficiente", donde la fuente principal de información es el internet y amigos $\mathrm{y}$ luego los padres (13); esto puede deberse al medio de obtención de la información debido a la tergiversación de conocimientos como consecuencia de la variedad y poca confiabilidad de las páginas visitadas.

En relación al nivel de conocimiento en la dimensión psicológica, se observa un NC medio con
41,2\%, resultado similar al estudio de Carranza M, Cahuana E, Fuentes W, Matta H, Pérez R y Zamudio R. (15); en ambos estudios debe analizarse que la población es de similar edad pero de diferentes zonas de Lima, como Lima Norte. Cabe resaltar que el NC en salud sexual y sus dimensiones no aumenta debido a la falta de orientación hacia un comportamiento basado en el juicio crítico para el bienestar del adolescente a pesar de la madurez del pensamiento que posea cada uno, normas y valores personales.

En relación al estudio realizado por Litano, se identificó que los adolescentes presentan prácticas de autocuidado inadecuadas (16); mientras que, el presente estudio halló que los adolescentes presentaron una capacidad de autocuidado media, a pesar que los estudios han sido realizados en diferentes años y con poblaciones diferentes en cantidad, los resultados se asemejan. Se puede decir que, con el transcurrir del tiempo se da mayor importancia a las intervenciones para contribuir con el autocuidado en salud sexual. En base a lo obtenido en el presente estudio se hace hincapié que los conocimientos sobre salud sexual deben ser impartidos con una base sólida a una temprana edad para poder fortalecer una buena toma de decisiones de los adolescentes en la prevención 
hacia la salud sexual (17) y tal como menciona Orem $\mathrm{D}$, el autocuidado es una actividad que el mismo individuo aprende en situaciones que se presentan en el transcurso de su vida, lo cual regularía las causas que puedan presentar un beneficio para su salud en base al propio desarrollo y funcionamiento (8).

Asimismo, se halló relación entre el $\mathrm{NC}$ y CA en salud sexual, resultados similar al de Santos M, Ferreira E y Ferreira M, quienes identificaron asociación entre el $\mathrm{NC}$ y las actitudes sobre salud sexual, donde la actitud se aborda como un comportamiento que se va formando a través de experiencias y otros comportamientos que se adoptan del entorno, estas actividades incluyen componentes cognitivos y afectivos (18). Estos resultados permiten determinar que, el conocimiento obtenido a través de las experiencias, buscan soluciones y prácticas de autocuidado según las experiencias; sin embargo, un mayor conocimiento no asegura necesariamente un comportamiento responsable porque el conocimiento por si solo como construcciones teóricas no es suficiente, es necesario demostrarlo con actitudes (8). Los hallazgos permiten identificar que a medida que aumenta el NC incrementa la CA en salud sexual de los adolescentes, es decir, existe una relación directa y positiva, ello se sustenta en lo postulado por Orem D, al señalar que para ejercer el autocuidado, primero se debe adquirir conocimientos y evaluarlos, ponerlos en práctica e ir aprendiendo de cada uno de ellos, con el fin de ser agentes del autocuidado; con los conocimientos que contribuyen al cuidado y/o a la mejora individual con ayuda de personas u otras fuentes de conocimiento, con el fin de mantener el desarrollo y bienestar personal (8).

De esta manera, la teoría de Orem D menciona la importancia de un buen autocuidado en cualquier situación que puedan presentar los adolescentes como conductas de riesgo en su salud y la toma de decisiones. Esta teoría orienta a los profesionales de Enfermería para poder brindar un cuidado preventivo (19), empleando un enfoque de atención integral en la salud de los adolescentes, en relación a las variables del estudio y sus respectivas dimensiones, estas son herramientas para que el equipo multidisciplinario de salud personalice actividades enfocándose en otras áreas diferentes a la biológica, según las necesidades de los adolescentes, para así fortalecer la toma de decisiones que promuevan su autocuidado.

Definitivamente, debe incrementarse en su abordaje la salud sexual del adolescente a través de intervenciones de promoción y prevención, para poder disminuir conductas de riesgo y fortalecer la tomas de decisiones en los adolescentes quienes necesitan fortalecer una serie de habilidades como la comunicación, interactuar, negociar, saber responder frente a la presión de compañeros, expresar sentimientos y pensamientos, evaluar opiniones y confrontar los conflictos. En cuanto a las limitaciones cabe mencionar la necesidad que se tuvo que adecuar los instrumentos a la realidad de la población de estudio y luego fueron validados mediante juicio de expertos y una prueba piloto. Asimismo, se recomienda realizar estudios posteriores con mayor amplitud y actualización en los instrumentos para un mejor abordaje de las variables.

\section{CONCLUSIONES}

El NC en salud sexual de los adolescentes en las dimensiones biológica y social se presenta con un nivel bajo y en la dimensión psicológica con un nivel medio.

La CA en salud sexual de los adolescentes en las dimensiones actividad y reposo y bienestar personal se presentan con una CA media y en las dimensiones interacción social, promoción del funcionamiento y desarrollo dentro de grupos sociales es alta.

Existe relación estadísticamente positiva y directa entre las variables nivel de conocimiento y capacidad de autocuidado en salud sexual de los adolescentes.

\section{Correspondencia:}

Aurora Marlene Giraldo Giraldo

Correo electrónico: aurora.giraldo.g@upch.pe

\section{REFERENCIAS BIBLIOGRAFICAS}

1. Organización Mundial de la Salud. Desarrollo en la adolescencia. Ginebra, Suiza: Organización Mundial de la Salud; 2019. (Citado 19 de abril del 2019). Disponible en: https://www.who.int/maternal_child_ adolescent/topics/adolescence/dev/es/.

2. Organización Mundial de la Salud. La salud sexual y su relación con la salud reproductiva: un enfoque operativo. Ginebra, Suiza: Organización Mundial de la Salud;2018(Citado 19 de abril del2019). Disponible en: https://www.who.int/ reproductivehealth/publications/sexual_health/shlinkages-rh/es/.

3. Ministerio de Salud. Modelo de Atención Integral de Salud Basado en Familia y Comunidad. Lima: 
Ministerio de Salud; 2011. (Citado 19 de abril del 2019). Disponible en: https://determinantes.dge.gob. pe/archivos/1880.pdf.

4. Ministerio de Educación.Aprendemos sobre sexualidad para orientar a nuestras hijas e hijos. Lima: MINEDU; 2010. (Citado 19 de abril del 2019). Disponible en: http://tutoria.minedu.gob.pe/assets/ aprendemos-sobre-sexualidad-para-orientarnuestras-hijas-hijos-actual.pdf.

5. Ministerio de Salud. Salud escolar. Lima: MINSA; 2019. (Citado 19 de abril del 2019). Disponible en: en: https://www.gob.pe/institucion/minsa/ campa $\% \mathrm{C} 3 \%$ Blas/89-salud-escolar

6. Dirección General de Salud de las personas. Salud sexual y reproductiva. Lima: Dirección General de Salud de las personas; 2015. (Citado 19 de abril del 2019). Disponible en: https://www.minsa.gob.pe/ portalweb/06prevencion/prevencion 2.asp?sub5 $=10$.

7. Antialón CA. Nivel de conocimiento sobre sexualidad en adolescentes de educación secundaria.Tesis. Lima: Universidad Peruana Cayetano Heredia; 2009. 50 p.

8. Orem D. Normas prácticas en enfermería. 1st ed. Madrid: Pirámide; 1983.

9. Leiva V, Cubillo K, Porras Y et al. Validación de apariencia, contenido y consistencia interna de la Escala de Apreciación de Agencia de Autocuidado (ASA) para Costa Rica, segunda versión en español para población conocida sana. Enfermería Actual en Costa Rica. 2016; (30). (Citado 19 de abril del 2019). Disponible en: https://www.redalyc.org/ articulo.oa? id $=44843206002$

10. Rojas R. Relación entre conocimientos sobre salud sexual y las conductas sexuales de riesgo en adolescentes de secundaria. I.E.M. Nuestra Señora de Montserrat. Lima 2010.Tesis. Lima: Universidad Nacional Mayor de San Marcos; 2011. 109 p.

11. Gallegos CE. Validez y confiabilidad de la versión en español de la escala: Valoración de las capacidades de Auto-cuidado. Desarrollo científico de Enfermería. 1998; 6 (9): 260-266.

12. Instituto Nacional de Estadística e Informática. Perú: perfil sociodemográfico. Lima, Perú: INEI; 2018 (Citado 19 de abril del 2019). Disponible en: https:// www.inei.gob.pe/media/MenuRecursivo/ publicaciones_digitales/Est/Lib1539/libro.pdf.
13. Dutt S, Manjula M. Sexual knowledge, attitude, behaviors and sources of influences in Urban college youth: A study from India. Indian Journal of Social Psychiatry. 2017; 33(4): 319-325. (Citado 19 de abril del 2019). Disponible en: http://www.indjsp.org/ article. asp? issn $=0971-9962$; year $=2017$; volumen $=33$; issue $=4 ;$ spage $=319$; epage $=326$; aulast $=$ Dutt .

14. Cardona D, Ariza A, Gaona C, Medina O. Conocimientos sobre sexualidad en adolescentes escolares en la ciudad de Armenica. Colombia. AMC. 2015 ; 19 (6): 568-576. (Citado 19 de abril del 2019). Disponible en: http://scielo.sld.cu/scielo.php?acript= sci_arttext\&pid=SIO2502552015000600003\&Ing= es.

15. Carranza E, Cahuana E, Fuentes W, Matta H, Pérez R, Zamudio R. Nivel de conocimientos sobre sexualidad en los adolescentes de $3^{\circ} \mathrm{a} 5^{\circ}$ de secundaria de una institución educativa periurbana en Lima Norte. Revista de Ciencia y Arte de Enfermería 2016; 1(1): 18-23. (Citado 19 de abril del 2019). Disponible en: http://www.cienciaenfermeria.org/index.php/ $\mathrm{rcae} / \mathrm{search} /$ search?simpleQuery=Nivel $+\mathrm{de}+$ conocim ientos + sobre + sexualidad + en + los + adolescentes $+\mathrm{de}+$ $3 \% \mathrm{C} 2 \% \mathrm{~B} 0+\mathrm{a}+5 \% \mathrm{C} 2 \% \mathrm{~B} 0+\mathrm{de}+$ secundaria $+\mathrm{de}+$ una + instituci $\% \mathrm{C} 3 \% \mathrm{~B} 3 \mathrm{n}+$ educativa + periurbana + en + Lima + Norte.\&searchField=query.

16. Litano VM. Prácticas de Autocuidado en Adolescentes de la I.E. "Virgen del Carmen" No 6014 VMT-Lima 2014. Tesis. Lima: Universidad Nacional Mayor de San Marcos; 2014. 89 pp.

17. Alligood M, Marriner A. Teoria del déficit de autocuidado. En: Berbiglia V. Banfield Modelos y teorías de enfermería. España: Elsevier; 2011. p. 265-281.

18. Santos MJ, Ferreira E, Ferreira M. Knowledge of and attitudes toward sexual and reproductive health among college students. España: Elsevier; 2016. (Citado 19 de abril del 2019). Disponible en: http://www.elsevier.es/es-revista-atencion-primaria27-articulo-knowledge-attitudes-toward-sexualreproductive-X0212656716593728

19. Naranjo Y, Concepción JA, Rodríguez M. La teoría Déficit de autocuidado: Dorothea Elizabeth Orem. Gac Méd Espirit. 2017; 19(3): 89-100. 